\title{
SPECTRAL PROPERTIES OF A CERTAIN CLASS OF COMPLEX POTENTIALS
}

\author{
BY \\ V. GUILLEMIN AND A. URIBE ${ }^{1}$
}

\begin{abstract}
In this paper we discuss spectral properties of the Schroedinger operator $-\Delta+q$ on compact homogeneous spaces for certain complex valued potentials $q$. We show, for instance, that for these potentials the spectrum of $-\Delta+q$ is identical with the spectrum of $-\Delta$.
\end{abstract}

Introduction. Let $G$ be a compact Lie group and $\Delta$ the bi-invariant LaplaceBeltrami operator on $G$. Fegan observed in $[\mathbf{F}]$ that there exist functions $q$ on $G$ for which the Schroedinger operator $-\Delta+q$ possesses an infinite sequence of eigenvalues $\lambda_{1}, \lambda_{2}, \ldots$ such that each $\lambda_{i}$ is also an eigenvalue of $-\Delta$. In this paper we will show that (a) potentials with this property exist not just on compact Lie groups but on arbitrary compact homogeneous spaces as well, and (b) for such potentials the entire spectrum of $-\Delta+q$ is identical with the spectrum of $-\Delta$.

In Fegan's examples as well as in ours, the potentials $q$ are complex-valued. This means that the operator $-\Delta+q$ is not selfadjoint. Fortunately, however, there exists a rather nice spectral theory for such operators. For instance their generalized eigenspaces are finite dimensional and span $L^{2}$. (See $\$ 2$ below.) In view of this we were surprised to find relatively little material in the literature on such operators. (A notable exception is Peter Sarnack's beautiful paper on Schroedinger operators with quasiperiodic $q$ 's. See $[\mathbf{S}]$.)

We will describe and sketch the proof of our main result. Let $G$ be a compact connected Lie group, $H$ a closed subgroup and $X$ the homogeneous space $G / H$. For simplicity we will assume for the moment that $G$ is semisimple (though this assumption is not essential; see $\S 4$ for the case $G=S^{1}$ ). Let $\hat{G}$ be the unitary dual of $G$. We can order $\hat{G}$ lexicographically by choosing a system of positive weights. Consider the Hilbert-space direct sum

$$
L^{2}(X)=\sum H_{\alpha}, \quad \alpha \in \hat{G},
$$

$H_{\alpha}$ being the $G$-invariant subspace of $L^{2}(X)$ on which $G$ acts according to the representation $\alpha$. Each irreducible subspace of $H_{\alpha}$ possesses a unique maximal weight vector. Let $H_{\alpha}^{+}$be the subspace of $H_{\alpha}$ spanned by these vectors and let

$$
L^{2}(X)^{+}=\sum H_{\alpha}^{+} \text {. }
$$

Received by the editors August 18, 1982 and, in revised form, November 19, 1982.

1980 Mathematics Subject Classification. Primary 58G25; Secondary 35P99.

'Research supported by the National University of Mexico.

(C) 1983 American Mathematical Society $0002-9947 / 83 \$ 1.00+\$ .25$ per page 
We will say that a smooth function $q$ is a Fegan potential if it belongs to $L^{2}(X)^{+}$and is of mean zero: $\int_{X} q d x=0$.

Now let $\Delta$ be the Laplace-Beltrami operator on $X$ associated with the killing form of $G$ and consider $-\Delta+q$ restricted to $L^{2}(X)^{+}$. The first term preserves the decomposition (1.2) whereas the second term shifts the $H_{\alpha}^{+}$'s to the right; so if we choose a basis for $L^{2}(X)^{+}$whose elements belong to the $H_{\alpha}^{+}$'s, the matrix for $-\Delta+q$ with respect to this basis is upper triangular and its diagonal entries are the eigenvalues of $-\Delta$; so

$$
\operatorname{Spec}(-\Delta+q)=\operatorname{Spec}(-\Delta) \quad \text { on } L^{2}(X)^{+} .
$$

Next let $g$ be the Lie algebra of $G, g^{C}$ its complexification and $n$ the maximal nilpotent subalgebra of $\mathrm{g}^{C}$ spanned by the positive root spaces. Every element $\xi \in \mathfrak{n}$ corresponds to a complex vector field $\hat{\xi}$ on $X$. We will denote by $D_{\xi}$ the operator "differentiation by $\hat{\xi}$ ". It is clear that

$$
L^{2}(X)^{+}=\bigcap_{\xi \in \mathfrak{n}} \operatorname{Ker}\left(D_{\xi}\right)
$$

and the $D_{\xi}$ 's commute with $-\Delta+q$. Therefore, if $\lambda \in \operatorname{Spec}(-\Delta+q)$ and $H_{\lambda}$ is the (finite-dimensional) generalized eigenspace associated with $\lambda, D_{\xi}$ maps $H_{\lambda}$ into itself; so we get a representation of $\mathfrak{n}$ on $H_{\lambda}$. By Lie's theorem (see [ $\left.\mathbf{J}\right]$ ), there exists a nonzero vector $v \in H_{\lambda}$ such that $D_{\xi} v=0$ for all $\xi \in \mathfrak{n}$. Thus by (1.3), $H_{\lambda} \cap L^{2}(X)^{+}$ $\neq 0$ and so, by the previous argument, $\lambda$ is an eigenvalue of $-\Delta$, proving that $\operatorname{Spec}(-\Delta+q)=\operatorname{Spec}(-\Delta)$. It is easy to see, by the way, that the dimension of $H_{\lambda}$ is equal to the multiplicity of $\lambda$ as an eigenvalue of $-\Delta$ (see $\S 2$ ).

We will conclude this section with an outline of the remainder of the paper. In $\S 2$ we will insert the few details needed in the proof above to make it rigorous. In $\$ 3$ we will examine briefly the inverse question: Given a complex potential $q$ on $X$ such that $\operatorname{Spec}(-\Delta+q)=\operatorname{Spec}(-\Delta)$, is $q$, up to translation by an element of $G$, a Fegan potential? One can define spectral invariants for complex potentials using the heat and wave equations associated with $-\Delta+q$ just as for real potentials, and our hope is that the vanishing of all these invariants will imply that $q$ is a Fegan potential. Here we restrict ourselves to rank one symmetric spaces and describe one such set of invariants in detail: the analogue for complex potentials of the "band invariants" of Weinstein [W].

In $\$ 4$ we discuss the theory of Fegan potentials on $S^{1}$. Such potentials are just functions in the positive Hardy space. We will prove directly that for such functions, $L^{2}\left(S^{1}\right)$ decomposes into an invariant direct sum $L^{2}(X)=C+\sum_{n \neq 0} H_{n}$ such that each $H_{n}$ is two dimensional and $\left(-\Delta+q-n^{2}\right)^{2} H_{n}=0$. We will say that $q$ is a $k$-band potential if it is even and if $\left(-\Delta+q-n^{2}\right) H_{n}$ is nonzero for $n \leqslant k$ and zero for $n>k$. We will prove a number of facts about the set of all $k$-band Fegan potentials. For example we will show it is a complex manifold equal to $C^{k}$ with $k$ rational hyperplanes deleted.

We suspect that there is no analogue of this finite band phenomenon in higher dimensions, the reason being the following: Let $\operatorname{Spec}(-\Delta)=\operatorname{Spec}(-\Delta+q)=\left\{\lambda_{1}\right.$, $\left.\lambda_{2}, \ldots\right\}$. Let $H_{i}^{0}$ and $H_{i}$ be the eigenspaces of $-\Delta$ and $-\Delta+q$ corresponding to $\lambda_{i}$. 
By (1.3) these spaces are modules with respect to the nilpotent Lie algebra $n$. It turns out that they are not only isomorphic as vector spaces, but modules as well, and the fact that $-\Delta+q$, restricted to $H_{i}$, is a module morphism imposes some severe restrictions on what it can be. In $\$ 5$ we will show that we can compute explicitly $-\Delta+q$ on the $H_{i}$ 's for $X=S^{2}$ by exploiting this fact.

We would like to thank Alan Weinstein for some timely suggestions about the material in \$2. We would also like to thank David Mumford and Henry McKean for elucidating for us the connection between the usual finite band potentials of the $\mathrm{KdV}$ theory and the finite band potentials described above.

2. Complex potentials. Let $H$ be a Hilbert space and $A$ an unbounded selfadjoint operator whose domain of definition is a dense subspace of $H$. We will assume that for some (and hence all) $\lambda \notin \operatorname{Spec}(A),(\lambda-A)^{-1}$ is completely continuous; and, in addition, we will assume that for some integer $n,(\lambda-A)^{-n}$ is of trace class. We will need the following theorem of Gohberg and Krein (see [G-K, Chapter V, §10]).

TheOREM. Let $T$ be a bounded operator and let $B=A+T$. Then:

(i) The spectrum of $B$ is discrete and has only $\pm \infty$ as points of accumulation.

(ii) For every $\varepsilon>0$ all but finitely many of the eigenvalues of $B$ lie in the sectors $-\varepsilon<\arg \lambda<\varepsilon$ and $\pi-\varepsilon<\arg \lambda<\pi+\varepsilon$.

(iii) Let $\lambda \in \operatorname{Spec}(B)$. Then the generalized eigenspace of $B$ associated with $\lambda$, i.e. the set of all $v \in H$ such that $(B-\lambda)^{k} v=0$ for some $k$, is finite dimensional.

(iv) The generalized eigenspaces span $H$.

If $X$ is a compact Riemannian manifold, $\Delta$ the Laplace-Beltrami operator and $q$ a smooth complex-valued function, we can apply this theorem to $A=-\Delta$ and $T=$ multiplication by $q$ to conclude

TheOREM 2.1. The spectrum of $-\Delta+q$ is discrete and has only $+\infty$ as a point of accumulation. If $\varepsilon>0$, all but finitely many of the eigenvalues of $-\Delta+q$ lie in the sector $-\varepsilon<\arg \lambda<\varepsilon$. Furthermore, its generalized eigenspaces are finite dimensional and span $H$.

Now let $G$ be a compact, connected semisimple Lie group, $H$ a closed subgroup of $G$ and $X=G / H$. Let $\Delta$ be the Laplace-Beltrami operator on $X$ associated with the killing form of $g$ and let $q$ be a Fegan potential. We will prove

THEOREM 2.2. $\operatorname{Spec}(-\Delta)=\operatorname{Spec}(-\Delta+q)$. Moreover for each $\lambda \in \operatorname{Spec}(-\Delta)$ the dimension of the generalized eigenspace of $-\Delta+q$ corresponding to $\lambda$ is equal to the dimension of the eigenspace of $-\Delta$ corresponding to $\lambda$.

Proof. As in $\S 1$ let $L^{2}(X)^{+}$be the subspace of $L^{2}(X)$ spanned by the maximal weight vectors. If $q$ and $f$ are in this space and $q$ is smooth, then by (1.3) $q f$ is in this space; so $-\Delta+q$ leaves this space invariant. If we apply the Gohberg-Krein theorem to the restriction of $-\Delta+q$ to $L^{2}(X)^{+}$, we conclude that the generalized eigenspaces of $-\Delta+q$ are finite dimensional and span $L^{2}(X)^{+}$. Let $\lambda$ be an eigenvalue of $-\Delta+q$ on $L^{2}(X)^{+}$and $f$ the corresponding eigenfunction. Decompose $f$ and $q f$ into 
their components with respect to the direct sum (1.2); i.e. let

$$
f=\sum f_{\alpha} \text { and } q f=\sum(q f)_{\alpha}, \quad \alpha \in \hat{G},
$$

where $f_{\alpha}$ and $(q f)_{\alpha}$ are in $H_{\alpha}$. Let $f_{\alpha_{0}}$ and $(q f)_{\alpha_{1}}$ be the leading terms in the series above with respect to the lexicographical ordering of $\hat{G}$. Since $q$ is of mean zero, $\alpha_{1}$ is strictly greater than $\alpha_{0}$ with respect to this ordering; so if we compare coefficients in the equation $(-\Delta+q) f=\lambda f$ we get $-\Delta f_{\alpha_{0}}=\lambda f_{\alpha_{0}}$, i.e. $\lambda \in \operatorname{Spec}(-\Delta)$. This shows that on $L^{2}(X)^{+}$, the spectrum of $-\Delta$ contains the spectrum of $-\Delta+q$.

Now let us pass from $L^{2}(X)^{+}$to $L^{2}(X)$. We will need

LEMma. Let $f \in L^{2}(X)$ be a common eigenfunction of the $D_{\xi}$ 's, $\xi \in \mathrm{n}$. Then $f \in L^{2}(X)^{+}$.

Proof. If $H$ is a vector space on which the group $G$ acts irreducibly, this lemma is well known. Now write $f$ as an infinite series in which each term lies in an irreducible $G$-invariant subspace of $L^{2}(X)$. Q.E.D.

To prove the theorem let $\lambda$ be an eigenvalue of $-\Delta+q$ and let $H_{\lambda}$ be the corresponding generalized eigenspace. By Theorem $2.1, H_{\lambda}$ is finite dimensional, and by (1.3) there is a natural representation of the nilpotent Lie algebra $n$ on $H_{\lambda}$; so by Lie's theorem there exists an $f \in H_{\lambda}$ which is a common eigenfunction of the $D_{\xi}$ 's. By the lemma, $f \in L^{2}(X)^{+}$, so $\lambda \in \operatorname{Spec}(-\Delta)$. This proves that $\operatorname{Spec}(-\Delta+q) \subset$ $\operatorname{Spec}(-\Delta)$. We still have to prove that if $\lambda$ is an eigenvalue of $-\Delta$ with multiplicity $m$, it is a generalized eigenvalue of $-\Delta+q$ with multiplicity $m$. To see this, let $D$ be a small disk in the complex plane centered about $\lambda$ and containing no other eigenvalue of $-\Delta$. Let $A_{t}$ be the operator $-\Delta+t q, 0 \leqslant t \leqslant 1$. Since $t q$ is a Fegan potential, $D$ contains no eigenvalue of $A_{t}$ either, except possibly $\lambda$; so if $\Gamma=\partial D$,

$$
P_{t}=\frac{1}{2 \pi i} \int_{\Gamma} \frac{d \lambda}{\left(\lambda-A_{t}\right)}
$$

is well defined and depends continuously on $t$. The trace of $P_{t}$ is the multiplicity of $\lambda$ as a generalized eigenvalue of $A_{t}$; so this multiplicity is the same for all $t$. Hence $\lambda \in \operatorname{Spec}(-\Delta+q)$ and has the same multiplicity as for $-\Delta$.

3. The band invariants. In this section we restrict ourselves to the case of rank-one symmetric space and describe the analogue for complex potentials of the theory of band asymptotics of Weinstein [W].

Let $X$ be a rank-one symmetric space, and let $q \in C^{\infty}(X)$ be a complex-valued function. Let $\Xi$ be the Hamiltonian vector field on $T^{*} X-0$ corresponding to the norm function arising from the Riemannian structure. The integral curves of $\Xi$, when projected onto $X$, are precisely the geodesics parametrized by arc length. Since $X$ is a rank-one symmetric space, these curves are all periodic with a common period $T$. We can then prove the following averaging lemma.

LEMMA 3.1. There exist zeroth order peusodifferential operators $F$ and $Q$ such that $F$ is invertible, $Q$ commutes with $\Delta$ and

$$
F(-\Delta+q) F^{-1}=-\Delta+Q \text {. }
$$


Moreover, the principal symbol of $Q$ is the function

$$
q^{\mathrm{av}}=\frac{1}{T} \int_{0}^{T}(\exp t \Xi)^{*}(q) d t
$$

The proof is the same as for real potentials, and we refer the reader to [G]. The spectrum of $-\Delta$ is of the form

$$
\lambda_{k}=\alpha k^{2}+\tau k, \quad k=0,1,2, \ldots
$$

each eigenvalue occurring with multiplicity $d_{k}=O\left(k^{n-1}\right)$ (see [B-G-M]). If $V_{k}$ is the eigenspace of $-\Delta$ corresponding to $\lambda_{k}$, then the operator $Q$ of Lemma 3.1 maps $V_{k}$ into itself. We let $\lambda_{1}^{(k)}, \ldots, \lambda_{d_{k}}^{(k)}$ be the eigenvalues of $Q$ restricted to $V_{k}$. Then Lemma 3.1 implies that the spectrum of $-\Delta+q$ consists of the points $\lambda_{k}+\lambda_{j}^{(k)}, j=1, \ldots, d_{k}$, $k=0,1,2, \ldots$.

We can now state the main result of the theory of "band asymptotics" for complex potentials.

THEOREM 3.2. Let $f$ be a function which is holomorphic on the spectrum of $Q$. Then there exist constants $\beta_{l}(f) \in C, l=0,1, \ldots$, depending linearly on $f$, such that

$$
\sum_{j=1}^{d_{k}} f\left(\lambda_{j}^{(k)}\right) \sim(2 \pi)^{-n} \sum_{l=0}^{\infty} k^{n-1-l} \beta_{l}(f)
$$

as $k \rightarrow \infty$. Moreover,

$$
\beta_{0}(f)=\int_{Z}\left(f \circ q^{\mathrm{av}}\right) d v
$$

where $Z$ is the unit cosphere bundle of $X$ and dv the canonical volume form on $Z$.

We sketch the proof of this theorem. Since $f$ is holomorphic on the spectrum of $Q$, the operator $f(Q)$ is well defined and is a zeroth order pseudofifferential operator with principal symbol $f \circ q^{\text {av }}$. Moreover, by (3.1), the operator $A$ which equals "multiplication by $k$ " on $V_{k}$ is an elliptic first-order selfadjoint pseudodifferential operator. For both of these statements we refer the reader to [Se]. With these operators at hand, one obtains (3.2) by analyzing the singularity of the trace of $f(Q) \exp (i t A)$ at $t=0$. For details see [W] or [U].

In the case of real potentials, the operator $Q$ of Lemma 3.1 is selfadjoint, and hence for each $f \in C^{\infty}(\mathbf{R})$ the operator $f(Q)$ is a well-defined pseudodifferential operator. Hence, in this case, the band invariants $\beta_{l}$ are compactly supported distributions on the real line. We would like to stress the fact that in the case of complex potentials, however, the band invariants are complex-analytic functionals.

We now turn to the question of when a complex potential $q$ is such that the spectrum of $-\Delta+q$ equals the spectrum of $-\Delta$. We would like to prove that this happens only when $q$ is a Fegan potential. According to Theorem 3.2, a necessary condition for a potential $q$ to be isospectral with zero is that all the band invariants 
should vanish when evaluated on functions vanishing at zero. In particular, we have

Proposition 3.3. Suppose that $\operatorname{Spec}(-\Delta+q)=\operatorname{Spec}(-\Delta)$. Then for all $r=1,2, \ldots$,

$$
\int_{Z}\left(q^{\mathrm{av}}\right)^{r} d v=0
$$

Unfortunately, at least for $S^{2}$, using the inverse of the Radon transform one can easily construct potentials $q$ satisfying (3.3) that are not Fegan potentials.

4. Fegan potentials on $S^{1}$. On $S^{1}$ Fegan potentials are just smooth functions belonging to the positive Hardy space; i.e. $q$ is a Fegan potential if its Fourier series is of the form

$$
q(\theta)=\sum_{n>0} b_{n} e^{i n \theta}
$$

We will give below a direct and fairly elementary proof of Theorem 2.2 for such potentials. However, we will first give an intuitive explanation of why this theorem is true, based on ideas from the Korteweg-DeVries theory. Let $X$ be the space of all smooth real-valued functions on $S^{1}$ whose zeroth Fourier coefficient is zero. This space can be given a symplectic structure by setting

$$
\Omega\left(q_{1}, q_{2}\right)=\int q_{1} Q_{2} d \theta
$$

for $q_{1}, q_{2} \in X$ and $Q_{2}$ an antiderivative of $q_{2}$. Let $L_{i}=L_{i}(q)$ be the "Lax invariants". These are functions on $X$ which can be written as integrals over $S^{1}$ of polynomial functions in $q$ and its derivatives. For instance the first three of these invariants are

$$
\begin{aligned}
& L_{1}(q)=\int \frac{q^{2}}{2} d \theta, \quad L_{2}(q)=\int\left(\frac{q_{x}^{2}}{2}+q^{3}\right) d \theta, \\
& L_{3}(q)=\int\left(\frac{q_{x x}^{2}}{5}+2 q\left(q_{x}\right)^{2}+q^{4}\right) d \theta .
\end{aligned}
$$

These functions have the following properties:

(I) they are in involution with respect to the symplectic structure (4.2); and

(II) the Hamiltonian flows generated by them are isospectral. Specifically, if $q_{1}$ and $q_{2}$ lie on the same trajectory of the Hamiltonian vector field associated with one of the $L_{i}$ 's, then the Schroedinger operators $-\Delta+q_{1}$ and $-\Delta+q_{2}$ have the same spectrum.

Now let $X^{C}$ be the complexification of the space $X$, i.e. the space of all complex-valued functions on $S^{1}$ with zero Fourier coefficient. Then (4.2) defines a complex symplectic form on $X^{C}$ and, by (4.3), the $L_{i}$ 's extend to holomorphic functions on $X^{C}$ and their Hamiltonian flows to holomorphic flows. The space $\Lambda$ of all $q$ 's satisfying (4.1) is a Lagrangian subspace of $X^{C}$ and, by (4.3), the $L_{i}$ 's vanish on $\Lambda$, so their Hamiltonian flows are tangent to $\Lambda$. If we could show that these flows act transitively on $\Lambda$ (or at least on a large open subset of $\Lambda$ ) we would have a very simple explanation of the isospectrality of the Fegan potentials. We will not attempt to make these ideas rigorous, but we will come back to the $\mathrm{KdV}$ picture later in this section. 
We will now give a direct proof of Theorem 2.2 for Fegan potentials on $S^{1}$. We will begin by considering the eigenvalue problem

$$
-\frac{d^{2}}{d \theta^{2}} f+q f=\lambda f
$$

for functions $f$ in the Hardy space. Extending $f$ and $q$ to holomorphic functions on the disk, (4.4) becomes

$$
(z(d / d z))^{2} f+q(z) f=\lambda f .
$$

This equation has a regular singular point at the origin, and its indicial equation is $\mu^{2}=\lambda$; so it is solvable if and only if $\lambda=n^{2}$ for an integer $n$, and in this case has a unique solution of the form $f(z)=z^{n} \tilde{f}(z)$, where $\tilde{f}$ is also holomorphic on the disk and $\tilde{f}(0)=1$.

Since $n^{2}$ is an eigenvalue of $-\Delta$ of multiplicity 2, we must find, in addition to $f$, a second eigenfunction (or generalized eigenfunction) for $-\Delta+q$ corresponding to $n^{2}$. We will assume this to be the restriction to $S^{1}$ of a meromorphic function on the disk of the form $g=z^{-n} h(z)$ where $h$ is holomorphic. We then require

$$
\left((z(d / d z))^{2}+q(z)-n^{2}\right) g=c f
$$

for some constant $c$ or, alternatively,

$$
(z(d / d z))^{2} h-2 n(z(d / d z)) h+q h=c z^{2 n} \tilde{f} .
$$

The indicial equation for (4.6) is $\mu(\mu-2 n)=0$, so it is always possible to find such an $h$ with $h(0)=1$. (The presence of $c$ on the right-hand side insures that we can solve (4.6) formally for the $z^{2 n}$ th coefficient of $h$.) Summarizing, we have proved that the spectrum of (4.4) consists of $n^{2}, n=0,1,2, \ldots$, and that except for $n=0$, there are, for each $n$, two independent generalized eigenfunctions. We leave it as an exercise to show that these functions span $L^{2}$.

We will next attempt to determine whether the eigenfunction $g$, obtained by solving (4.6), is simple or generalized. Let

$$
h(z)=1+a_{1} z+a_{2} z^{2}+\cdots
$$

and

$$
q(z)=b_{1} z+b_{2} z^{2}+\cdots .
$$

By (4.6) we can determine the $a_{i}$ 's uniquely from the $b_{i}$ 's for $i<2 n$. The $2 n$th term of $q h$ is

$$
b_{2 n}+b_{1} a_{2 n-1}+\cdots+b_{2 n-1} a_{1}
$$

and $c=0$ in (4.6) if and only if (4.9) is zero. But the vanishing of $c$ means that $g$ is an ordinary eigenfunction, so we have proved

Proposition 4.1. The vanishing of (4.9) is a necessary and sufficient condition for the nth eigenvalue of (4.4) to be a true double eigenvalue.

Let us describe this condition more explicity. For $0<k<2 n$ we have

$$
-k(k-2 n) a_{k}=b_{k}+b_{1} a_{k-1}+\cdots+b_{k-1} a_{1} .
$$


This gives us inductively, for $k<2 n$,

$$
a_{k}=P_{k, n}\left(b_{1}, \ldots, b_{k}\right) \text {, }
$$

where $P_{k, n}$ is a polynomial degree $k$ in the $b_{i}$ 's and is homogeneous of degree $k$ providing we weight $b_{1}$ with weight one, $b_{2}$ with weight 2 , etc. Substituting these values of $a_{k}$ into (4.9) we get

THEOREM 4.2. There exists a polynomial function $Q_{n}$ of the variables $b_{1}, \ldots, b_{2 n-1}$ such that $n^{2}$ is a genuine double eigenvalue of (4.4) if and only if $b_{2 n}=Q_{n}\left(b_{1}, \ldots, b_{2 n-1}\right)$. Moreover, $Q_{n}$ is weighted-homogeneous of degree $2 n$ providing we assign weight $i$ to the variable $b_{i}$ for each $i=1, \ldots, 2 n-1$.

REMARK. It is not hard to calculate explicitly the first few of the $Q_{n}$ 's. For instance,

$$
Q_{1}=-b_{1}^{2} \quad \text { and } \quad Q_{2}=-\left(\frac{2}{3} b_{1}, b_{3}+\frac{2}{3} b_{1}^{2} b_{2}+\frac{b_{1}^{4}}{36}+\frac{b_{2}^{2}}{4}\right) .
$$

In the usual $\mathrm{KdV}$ theory, one set of objects of considerable interest is the "finite band" potentials. By definition, an $n$-band potential is an even real-valued function on the circle such that the spectrum of $-\Delta+q$ has the following features. The first $2 n+1$ eigenvalues are simple, but from then on all eigenvalues are double. It has been shown that the set of all these potentials forms a finite-dimensional symplectic manifold on which the KdV flows act as a completely integrable system. (See, for instance, [McK-V].) Using Theorem 4.3, we will show that analogous results are true for finite hand Fegan potentials. We will say that a Fegan potential $q$ is an $n$-band potential if:

(i) it is even, i.e. $q(\theta)=q(\theta+\pi)$;

(ii) the eigenvalues $k^{2}, k=0,1, \ldots, n$, are strictly generalized eigenvalues;

(iii) the remaining eigenvalues are all genuine double eigenvalues.

The assumption that $q$ is even implies that its holomorphic extension to the disk is represented by a power series of the form

$$
q(z)=\sum_{k=1}^{\infty} b_{2 k} z^{2 k} .
$$

To obtain the set of all $n$-band potentials we can specify $b_{2}, \ldots, b_{2 n}$ arbitrarily, providing we make certain that

$$
b_{2 k} \neq Q_{k}\left(b_{2}, \ldots, b_{2 k-2}\right)
$$

for $k \geqslant n$, and then the conditions $b_{2 k}=Q_{k}\left(b_{2}, \ldots, b_{2 k-2}\right)$ for $k>n$ determine all the remaining coefficients of $q$. Thus we have proved

THEOREM 4.3. The set of all $n$-band Fegan potentials is isomorphic to $C^{n}$ with the $n$ rational hypersurfaces (4.12) deleted.

The Hamiltonian flows associated with the Lax functions map this set into itself. We will attempt here just to describe the flow associated with $L_{1}$. On the circle this Hamiltonian gives rise to the flow $q \rightarrow q_{t}$ for $t \in \mathbf{R}$ where $q_{t}(\theta)=q(t+\theta)$. If we 
expand $q$ into its Fourier coefficients as in (4.1) this becomes the one-parameter group

$$
b_{n} \rightarrow e^{i t n} b_{n}, \quad n+1,2, \ldots,
$$

with $t \in \mathbf{R}$; or, if we complexify this flow

$$
b_{n} \rightarrow \lambda^{n} b_{n}, \quad n=1,2, \ldots,
$$

with $\lambda \in C^{*}$. This explains why the $Q_{k}$ 's are weighted homogeneous with each $b_{i}$ weighted of degree $i$.

David Mumford has suggested to us a method for getting explicit closed-form expressions for the finite-band Fegan potentials. Consider the manifold of $n$-band potentials whose first $2 n+1$ eigenvalues are $\lambda_{0}, \ldots, \lambda_{2 n}$ where $\lambda_{0}=0$ and $\lambda_{2 k-1}=$ $k^{2}, k=1, \ldots, n$. From the Its-Matseev theory, one gets closed form expressions for these potentials. The limits of these expressions as $\lambda_{2 k} \rightarrow k^{2}$ for $k=1, \ldots, n$ should be the potentials above. Explicit expressions for these limits (corresponding to the operation of "band-pinching") can be found in [McK].

5. Fegan potentials on $S^{2}$. Let $q$ be a Fegan potential on the homogeneous space $X$. Let $\lambda_{1}, \lambda_{2}, \ldots$ be the eigenvalues of $-\Delta+q$ and let $H_{1}, H_{2}, \ldots$ be the corresponding generalized eigenspaces. By Theorem 2.2 the $\lambda_{i}$ 's and the dimensions of the $H_{i}$ 's are independent of $q$; so the only spectral data which does depend on $q$ is the string of matrices $J_{1}, J_{2}, \ldots$, where $J_{i}$ is the matrix representing $-\Delta+q$ on $H_{i}$ in a Jordan canonical basis. In this section we will show how to compute these matrices for even potentials on $S^{2}$.

We will denote by $x, y$ and $z$ the standard coordinate functions on $\mathbf{R}^{3}$ and also their restrictions to $S^{2}$. We recall the following standard facts about the Laplace operator $\Delta$ on $S^{2}$. (See, for instance, [We, pp. 61, 62].)

(1) The eigenvalues of $-\Delta$ are $r(r+1), r=0,1,2, \ldots$

(2) The eigenspace corresponding to $r(r+1)$ is $(2 r+1)$-dimensional and is spanned by the functions

$$
P_{r, k}(z)(x+i y)^{k}, \quad k=0, \ldots, r
$$

and their conjugates

$$
P_{r, k}(z)(x-i y)^{k}, \quad k=1, \ldots, r
$$

where

$$
P_{r, k}(z)=\left(1-z^{2}\right)^{-k}(d / d z)^{r-k}\left(1-z^{2}\right)^{r} .
$$

Note that (5.3) is a polynomial in $z$ of degree $r-k$.

(3) $S O(3)$ acts irreducibly on this space and its highest weight vector is $(x+i y)^{r}$.

From (3) one easily deduces

THEOREM 5.1. A smooth function $q$ is a Fegan potential on $S^{2}$ if and only if it is of the form $q(x+i y)$, where $q$ is a smooth function on the closed unit disk $D$ which is holomorphic on Int $D$ and zero at the origin. 
The space of these functions can also be characterized by (1.3). In the case $S O(3)$ the nilpotent algebra $\mathfrak{n}$ is one dimensional and spanned by the vector field $X+i Y$ where

$$
X=y \frac{\partial}{\partial z}-z \frac{\partial}{\partial y} \quad \text { and } \quad Y=z \frac{\partial}{\partial x}-x \frac{\partial}{\partial z}
$$

are the infinitesimal rotations about the $x$ and $y$ axes. Thus a smooth function $q$ is a Fegan potential if and only if

$$
(X+i Y) q=0 .
$$

We will henceforth restrict our attention to even potentials, i.e. potentials $q=$ $q(x, y, z)$ on $S^{2}$ for which $q(-x,-y,-z)=q(x, y, z)$. If $q$ is an even Fegan potential it can be written as a convergent power series of the form

$$
q(x+i y)=\sum_{n>0} b_{2 n}(x+i y)^{2 n} .
$$

Let $2 k$ be the order of the leading nonzero term in (5.5). Our main result is

THEOREM 5.2. Let $J_{r}$ be the $2 r+1$ by $2 r+1$ matrix having ones at all its superdiagonal entries and zeros at all other entries. Let $H_{r}$ be the rth generalized eigenspace of $-\Delta+q$. Then one can choose a basis for $H_{r}$ such that, in terms of this basis, $-\Delta+q-r(r=1)$ is represented by the matrix $J_{r}^{2 k}$.

From this theorem we immediately deduce

COROllary 5.3. Let $n_{r}$ be the degree of the minimal polynomial of $-\Delta+q$ on $H_{r}$. Then either $n_{r} \rightarrow \infty$ as $r \rightarrow \infty$ or $q$ is identically zero.

For the proof of Theorem 5.2 we will need a number of preliminary results. We first note that $X+i Y$, viewed as a differential operator on $L^{2}$, leaves $H_{r}$ fixed. We will need

Proposition 5.4. The minimal polynomial of $X+i Y$ on $H_{r}$ is $t^{2 r+1}$.

Proof. Consider $(X+i Y)^{s}$ as a differential operator on $L^{2}$. Its kernel is a closed subspace of $L^{2}$ which is invariant with respect to $-\Delta$ and $-\Delta+q$. By the results of $\S 2,-\Delta$ and $-\Delta+q$ have the same eigenvalues on this subspace, and the multiplicities of the eigenvalues are the same. But the multiplicity of the eigenvalues $r(r+1)$ of $-\Delta$ on this space equals $s$ if $s \leqslant 2 r+1$ and equals $2 r+1$ if $s \geqslant 2 r+1$, so the same is true of $-\Delta+q$. In particular, $H_{r}$ is contained in this space if $s \geqslant 2 r+1$ and not contained in it if $s \leqslant 2 r+1$. Q.E.D.

Let us denote by $K_{r}$ the $L^{2}$-closure of the space spanned by the set of all spherical harmonics of the form $P_{m, s}(z)(x+i y)^{s}$ where either $s=r$ and $m>r$ or $m \geqslant s>r$. (See (5.1) and (5.3).) It is clear that this space is invariant with respect to $-\Delta$ and $-\Delta+q($ for instance,

$$
P_{m, s}(z)(x+i y)^{s+k}=\sum_{j=0} a_{j} P_{s+k+j, s+k}(z)(x+i y)^{s+k}
$$

since $P_{r, s}(z)$ is a polynomial of degree $\left.r-s\right)$. We will need

Proposition 5.5. $-\Delta+q-r(r+1)$ is invertible on $K_{r}$. 
Proof. The spectrum of $-\Delta+q$ and the spectrum of $-\Delta$ are the same on $K_{r}$. But the spectrum of $-\Delta$ on $K_{r}$ consists of the eigenvalues $n(n+1), n>r$. In particular, it does not include $r(r+1)$. Q.E.D.

We will next show that Theorem 5.2 can be readily deduced from the following.

THEOREM 5.6. For $2 r \geqslant 2 k$ there exists a sequence of functions $f_{0}, \ldots, f_{2 k}$ in $H_{r}$ and $a$ nonzero constant $\lambda$ such that:

(a) $(X=i Y) f_{0}=0$ and $(X+i Y) f_{m}=f_{m-1}$ for $1 \leqslant m \leqslant 2 k$.

(b) $(-\Delta+q-r(r+1)) f_{m}=0$ for $m<2 k$.

(c) $(-\Delta+q-r(r+1)) f_{2 k}=\lambda f_{0}$.

To show that this theorem implies Theorem 5.2, let $J_{0}$ be the linear mapping of $H_{r}$ into itself associated with $X+i Y$, and let $A$ be the linear mapping associated with $-\Delta+q-r(r+1)$. By Proposition 5.4, $J_{0}$ is a nondegenerate nilpotent mapping, i.e. $J^{2 r} \neq 0$ and $J^{2 r+1}=0$, where $2 r+1=\operatorname{dim} H_{r}$. Since $A$ and $J_{0}$ commute, $A$ can be written as a polynomial function of $J_{0}$ with complex coefficients. By Theorem 5.6 this polynomial has to be of the form

$$
\begin{aligned}
A & =a_{2 k} J_{0}^{2 k}+\text { higher order terms in } J_{0} \\
& =a_{2 k} J_{0}^{2 k}\left(I+c_{1} J_{0}+c_{2} J_{0}^{2}+\cdots\right)
\end{aligned}
$$

where $a_{2 k} \neq 0$. Thus if we set

$$
J=\left(a_{2 k}\right)^{1 / 2 k} J_{0}\left(I+c_{1} J_{0}+c_{2} J_{0}^{2}+\cdots\right)^{1 / 2 k},
$$

the last factor on the right being defined by the power series for $(1+t)^{1 / 2 k}$, we obtain $A=J^{2 k}$. Finally, it is clear that $J$ is also a nondegenerate nilpotent mapping. Q.E.D.

We will construct only the $f_{m}$ 's in Theorem 5.6 for $r \geqslant 2 k$. (For $r<2 k \leqslant 2 r$ the construction is similar but somewhat more involved.) We will first show

LEMMA 5.7. There exist functions $g_{0}, \ldots, g_{2 k-1}$ in $H_{r}$ such that $g_{m}-P_{r, r-m}(z)$ $\cdot(x+i y)^{r-m}$ is in $K_{r+1}$ and $(-\Delta+q-r(r+1)) g_{m}=0$.

Proof. By (5.1),

$$
(-\Delta-r(r+1)) P_{r, r-m}(x+i y)^{r-m}=0 ;
$$

so $(-\Delta+q-r(r+1)) P_{r, r-m}(x+i y)^{r-m}$ is in $K_{r+1}$. By Proposition 5.5 there exists a function $h_{m} \in K_{r+1}$ such that

$$
(-\Delta+q-r(r+1)) P_{r, r-m}(x+i y)^{r-m}=(-\Delta+q-r(r+1)) h_{m} .
$$

Now set $g_{m}=P_{r, r-m}(x+i y)^{r-m}-h_{m}$. Q.E.D.

Next we will show

LEMMA 5.8. There exists a function $g_{2 k} \in H_{r}$ and a nonzero constant $\lambda$ such that $g_{2 k}-P_{r, r-2 k}(x+i y)^{r-2}$ is in $K_{r}$ and $(-\Delta+q-r(r+1)) g_{2 k}=\lambda g_{0}$.

We will deduce this from the following elementary fact about Legendre polynomials which we will prove in the appendix. 
LEMMA 5.9. There exist constants $c_{0}, c_{2}, \ldots, c_{2 k}$ with $c_{0} \neq 0$ such that

$$
P_{r, r-2 k}(z)=c_{0}+\sum_{m=1}^{k} c_{2 m} P_{r+2 m, r}(z) \text {. }
$$

To prove Lemma 5.8 we note that

$$
(-\Delta+q-r(r+1)) P_{r, r-2 k}(z)(x+i y)^{r-2 k}=b_{2 k} P_{r, r-2 k}(x+i y)^{r}
$$

modulo $K_{r}$, where $b_{2 k}$ is the coefficient of the leading nonzero term in (5.5). By Lemma 5.7, $g_{0}=(x+i y)^{r}$ modulo $K^{r}$; so by (5.6) we can express the right-hand side of (5.7) as $\lambda g_{0}$ plus an element of $K_{r}$ with $\lambda \neq 0$. In view of Proposition 5.5, this proves the lemma. Q.E.D.

Finally we will prove Theorem 5.6. We first observe that $X+i Y$, viewed as a linear mapping on the space of $r$ th order spherical harmonics, acts as a "shift" operator on the functions (5.1); i.e.

$$
(X+i Y) P_{r, r-m}(z)(x+i y)^{r-m}=\gamma P_{r, r-m+1}(x+i y)^{r-m+1},
$$

where $\gamma$ is a nonzero constant depending on $m$ and $r$. In addition, $X+i Y$ leaves the space $K_{r}$ fixed; so by Lemmas 5.7 and 4.8, $\gamma(X+i Y) g_{m}-g_{m-1} \in K_{r}$, but the term on the right is a generalized eigenfunction of $-\Delta+q-r(r+1)$, so it has to be zero by Proposition 5.5. Therefore, by setting the $f_{m}$ 's equal to appropriate nonzero constant multiples of the $g_{m}$ 's, we can arrange that they satisfy all the conditions of Theorem 5.6.

Appendix. We will prove here a technical lemma about Legendre functions which we used in $§ 5$, and for which we found no ready source in the literature. (See Lemma 5.9.) Let

$$
P_{r, k}(z)=\left(1-z^{2}\right)^{-k}(d / d z)^{r-k}\left(1-z^{2}\right)^{r}
$$

for $r \geqslant k . P_{r, k}$ is a polynomial of order $r-k$ whose leading term is nonzero, and is even or odd depending on whether $r-k$ is even or odd. In particular the polynomials $1, P_{*+2, r}, \ldots, P_{r+2 k, r}$ form a basis for the space of even polynomials of degree $\leqslant 2 k$. Now suppose $r \geqslant 2 k$. Since $P_{r, r-2 k}$ is an even polynomial of degree $2 k$, we can find constants $c_{0}, c_{2}, \ldots, c_{2 k}$ depending on $r$ and $k$ such that

$$
P_{r, r-2 k}=c_{0}+c_{2} P_{r+2, r}+\cdots+c_{2 k} P_{r+2 k, r} \text {. }
$$

We will prove

THEOREM. The coefficient $c_{0}$ in (A1) is nonzero.

Proof. We can rewrite (Al) in the form

$$
\left(1-z^{2}\right)^{2 k}(d / d z)^{2 k}\left(1-z^{2}\right)^{r}=c_{0}\left(1-z^{2}\right)^{r}+\cdots+c_{2 k}(d / d z)^{2 k}\left(1-z^{2}\right)^{r+2 k} \text {. }
$$

Integrating from -1 to 1 , we get, for $c_{0}$, the formula

$$
\begin{aligned}
c_{0} \int_{-1}^{1}\left(1-z^{2}\right)^{r} d z & =\int_{-1}^{1}\left(1-z^{2}\right)^{2 k}\left(\frac{d}{d z}\right)^{2 k}\left(1-z^{2}\right)^{r} d z \\
& =\int_{-1}^{1}\left(1-z^{2}\right)^{r}\left(\frac{d}{d z}\right)^{2 k}\left(1-z^{2}\right)^{2 k} d z=\int_{-1}^{1}\left(1-z^{2}\right)^{r} P_{2 k}(z) d z,
\end{aligned}
$$


where $P_{2 k}(z)$ is the standard Legendre function of degree $2 k$. Therefore, setting

$$
\gamma_{r, k}=\int_{-1}^{1}\left(1-z^{2}\right)^{r} P_{2 k}(z) d z
$$

we have to show that this integral is nonzero for $r \geqslant 2 k$. But, for $r>k$,

$$
(r-m)(2 r+2 k+1) \gamma_{r, k}=2 r^{2} \gamma_{r-1, k} .
$$

(See Whittaker and Watson [W-W, $\$ 15.211$, Example 6].) Thus $\gamma_{r, k} \neq 0$ for all $r>k$ providing $\gamma_{k, k} \neq 0$. Suppose $\gamma_{k, k}=0$. Then

$$
\int_{-1}^{1}\left(1-z^{2}\right)^{k} P_{2 k}(z) d z=0 .
$$

Therefore because of the orthogonality relations,

$$
\int_{-1}^{1} P_{k}(z) P_{m}(z) d z=0 \quad \text { for } k \neq m,
$$

$\left(1-z^{2}\right)^{k}$ is a linear combination of $P_{0}, P_{2}, \ldots, P_{2 k-2}$. However, this would imply that $\left(1-z^{2}\right)^{k}$ is of degree $<2 k$.

\section{REFERENCES}

[B-G-M] M. Berger, P. Gauduchon and E. Mazet, Le spectre d'une variété Riemanniene, Lecture Notes in Math., Vol. 194, Berlin and New York, 1971.

[F] H. Fegan, Special function potentials for the Laplacian, Canad. J. Math. 34 (1982), 1183-1194.

[G] V. Guillemin, Band asymptotics in two dimensions, Adv. in Math. 42 (1981), 248-282.

[G-K] I. C. Gohberg and M. G. Kreìn, Introduction to the theory of linear nonselfadjoint operators, Transl. Math. Monos, Vol. 18, Amer. Math. Soc., Providence, R. I., 1969.

[J] N. Jacobson, Lie algebras, Interscience, New York, 1962.

[McK] H. McKean, Integrable systems and algebraic curves, Global Analysis, Lecture Notes in Math., Vol. 755, Springer, Berlin, 1979.

[McK-V] H. McKean and P. Van Moerbeke, The spectrum of Hill's equation, Invent. Math. 30 (1975), 217-274.

[S] P. Sarnack, Spectral behavior of quasi-periodic potentials, Comm. Math. Phys. 84 (1982), 377-401.

[Se] R. T. Seeley, Complex powers of an elliptic operator, Singular Integrals, Proc. Sympos. Pure Math., Vol. 10, Amer. Math. Soc., Providence, R. I., 1967, pp. 288-307.

[U] A. Uribe, The averaging method and spectral invariants, Ph.D. Thesis, M.I.T., 1982.

[W] A. Weinstein, Asymptotics of eigenvalue clusters for the Laplacian plus a potential, Duke Math. J. 44 (1977), 883-892.

[We] H. Weyl, The theory of groups and quantum mechanics, Dover, New York, 1950

[W-W] E. T. Whittaker and G. N. Watson, A course in modern analysis, Cambridge Univ. Press, Cambridge, 1935.

Department of Mathematics, Massachusetts Institute of Technology, Cambridge, MasSACHUSETTS 02139 